\title{
RED AND DEAD: THE PROGENITOR OF SN 2012aw IN M95
}

\author{
M. Fraser ${ }^{1}$, J. R. Maund ${ }^{1,8}$, S. J. Smarti ${ }^{1}$, M.-T. Botticella ${ }^{2}$, M. DAll'Ora $^{2}$, C. Inserra ${ }^{1}$, L. Tomasella $^{3}$, \\ S. Benetti ${ }^{3}$, S. Ciroi ${ }^{3}$, J. J. Elddridge ${ }^{4}$, M. Ergon ${ }^{5}$, R. Kotak ${ }^{1}$, S. Mattila ${ }^{6}$, P. OChner ${ }^{3}$, \\ A. Pastorello ${ }^{3}$, E. Reilly $^{1}$, J. Sollerman $^{5}$, A. Stephens $^{7}$, F. Taddia $^{5}$, and S. Valenti ${ }^{3}$ \\ ${ }^{1}$ School of Mathematics and Physics, Queens University Belfast, Belfast BT7 1NN, UK; m.fraser@qub.ac.uk \\ ${ }^{2}$ INAF Osservatorio Astronomico di Capodimonte, Salita Moiariello 16, I-80131 Napoli, Italy \\ ${ }^{3}$ INAF Osservatorio Astronomico di Padova, vicolo dell Osservatorio 5, I-35122 Padova, Italy \\ ${ }^{4}$ Department of Physics, University of Auckland, Private Bag 92019, Auckland, New Zealand \\ ${ }^{5}$ The Oskar Klein Centre, Department of Astronomy, AlbaNova, Stockholm University, SE-10691 Stockholm, Sweden \\ ${ }^{6}$ Tuorla Observatory, Department of Physics and Astronomy, University of Turku, Väisäläntie 20, FI-21500 Piikkiö, Finland \\ ${ }^{7}$ Gemini Observatory, 670 North Aohoku Place, Hilo, HI 96720, USA \\ Received 2012 April 6; accepted 2012 September 20; published 2012 October 15
}

\begin{abstract}
Core-collapse supernovae ( $\mathrm{SNe}$ ) are the spectacular finale to massive stellar evolution. In this Letter, we identify a progenitor for the nearby core-collapse SN 2012aw in both ground-based near-infrared and space-based optical pre-explosion imaging. The SN itself appears to be a normal Type II Plateau event, reaching a bolometric luminosity of $10^{42} \mathrm{erg} \mathrm{s}^{-1}$ and photospheric velocities of $\sim 11,000 \mathrm{~km} \mathrm{~s}^{-1}$ from the position of the $\mathrm{H} \beta$ P-Cygni minimum in the early SN spectra. We use an adaptive optics image to show that the SN is coincident to within 27 mas with a faint, red source in pre-explosion $H S T+$ WFPC2, VLT+ISAAC, and NTT+SOFI images. The source has magnitudes $F 555 W=26.70 \pm 0.06, F 814 W=23.39 \pm 0.02, J=21.1 \pm 0.2, K=19.1 \pm 0.4$, which, when compared to a grid of stellar models, best matches a red supergiant. Interestingly, the spectral energy distribution of the progenitor also implies an extinction of $A_{V}>1.2 \mathrm{mag}$, whereas the $\mathrm{SN}$ itself does not appear to be significantly extinguished. We interpret this as evidence for the destruction of dust in the SN explosion. The progenitor candidate has a luminosity between 5.0 and $5.6 \log L / L_{\odot}$, corresponding to a zero-age main-sequence mass between 14 and $26 M_{\odot}$ (depending on $A_{V}$ ), which would make this one of the most massive progenitors found for a core-collapse SN to date.
\end{abstract}

Key words: galaxies: individual (M95) - supergiants - supernovae: general - supernovae: individual (SN2012aw)

Online-only material: color figures

\section{INTRODUCTION}

That massive red supergiants explode as Type IIP (Plateau) supernovae $(\mathrm{SNe})$ at the end of their lives has been clearly established (for example, Van Dyk et al. 2003; Smartt et al. 2004; Mattila et al. 2008; Fraser et al. 2011), and confirmed by the disappearance of their progenitors (Maund \& Smartt 2009). What remains of great interest, however, is understanding how SN progenitor properties (e.g., mass, radius, metallicity) correlate with the explosion characteristics (kinetic energy, synthesized ${ }^{56} \mathrm{Ni}$, luminosity etc). The continued identification of progenitors of nearby SNe, coupled with follow-up observations of the $\mathrm{SNe}$, can help shed light on this link. In this Letter we present an analysis of the progenitor of SN 2012aw, which is the coolest, and probably the most massive progenitor of a Type IIP SN found thus far.

SN 2012aw in M95 was discovered independently by several amateur astronomers (Fagotti et al. 2012), with a first detection on 2012 March 16.9. A non-detection at a limiting magnitude of $R \gtrsim 20.7$ (Poznanski et al. 2012) on March 15.3 sets a rigorous constraint on the age of the SN at discovery of $<1.6$ days, and we adopt an explosion epoch of March 16.0 UT ( \pm 0.8 days). Munari (2012) obtained a spectrum of the SN on March 17.8, which showed a featureless blue continuum; subsequent spectra (Itoh et al. 2012; Siviero et al. 2012) suggested the SN was a young Type IIP, although this classification is yet to be confirmed by the emergence of a plateau in the light curve at three to four weeks after explosion. A candidate progenitor for SN 2012aw

\footnotetext{
8 Royal Society Research Fellow.
}

was identified in archival Hubble Space Telescope (HST) data by Elias-Rosa et al. (2012) and Fraser et al. (2012), who both suggested that a faint red supergiant was the likely precursor star.

The host galaxy of SN 2012aw, M95, is a barred and ringed spiral galaxy with an inclination of $55^{\circ}$. Freedman et al. (2001) measured a Cepheid-based distance to M95 of $10 \mathrm{Mpc}$ (corresponding to $\mu=30.0 \pm 0.09 \mathrm{mag}$ ), which we adopt in all of the following. This distance is consistent with that obtained from the tip of the red giant branch (Rizzi et al. 2007). We also adopt a foreground (Milky Way) extinction of $E(B-V)=0.028 \mathrm{mag}$ from Schlegel et al. (1998). Using the line strengths for the closest $\mathrm{H}$ II region reported in McCall et al. (1985) we calculate a metallicity of $12+\log [\mathrm{O} / \mathrm{H}]=$ $8.8 \pm 0.1$ using the $\left[\mathrm{O}_{\mathrm{III}}\right] /\left[\mathrm{N}_{\mathrm{II}}\right]$ relation of Pettini \& Pagel (2004). From the recent study of the radial metallicity gradient in M95 by Pilyugin et al. (2006), at the position of SN 2012aw we estimate a metallicity of $12+\log [\mathrm{O} / \mathrm{H}]=$ $8.6 \pm 0.2$. Hence, we suggest that the metallicity of the progenitor of SN 2012aw is approximately solar (for comparison, Milky Way $\mathrm{H}$ II regions have a typical metallicity of $12+\log [\mathrm{O} / \mathrm{H}]=$ $8.7 \pm 0.3$; Hunter et al. 2009), albeit with the caveat that metallicity calibrations have significant uncertainties (as discussed in this context by Smartt et al. 2009).

SN 2012aw was detected in X-rays with the Swift X-Ray Telescope by Immler \& Brown (2012), at a luminosity of $L_{X}=9.2 \pm 2.5 \times 10^{38} \mathrm{erg} \mathrm{s}^{-1}$. This is at the upper end of the range of Type IIP X-ray luminosities $\left(0.16-4 \times 10^{38} \mathrm{erg} \mathrm{s}^{-1}\right.$; Chevalier et al. 2006), although still much lower than the typical values seen for Type IIn SNe $\left(0.2-1.6 \times 10^{41} \mathrm{erg} \mathrm{s}^{-1}\right.$; Fox et al. 2000) which are interacting with significant amounts of 

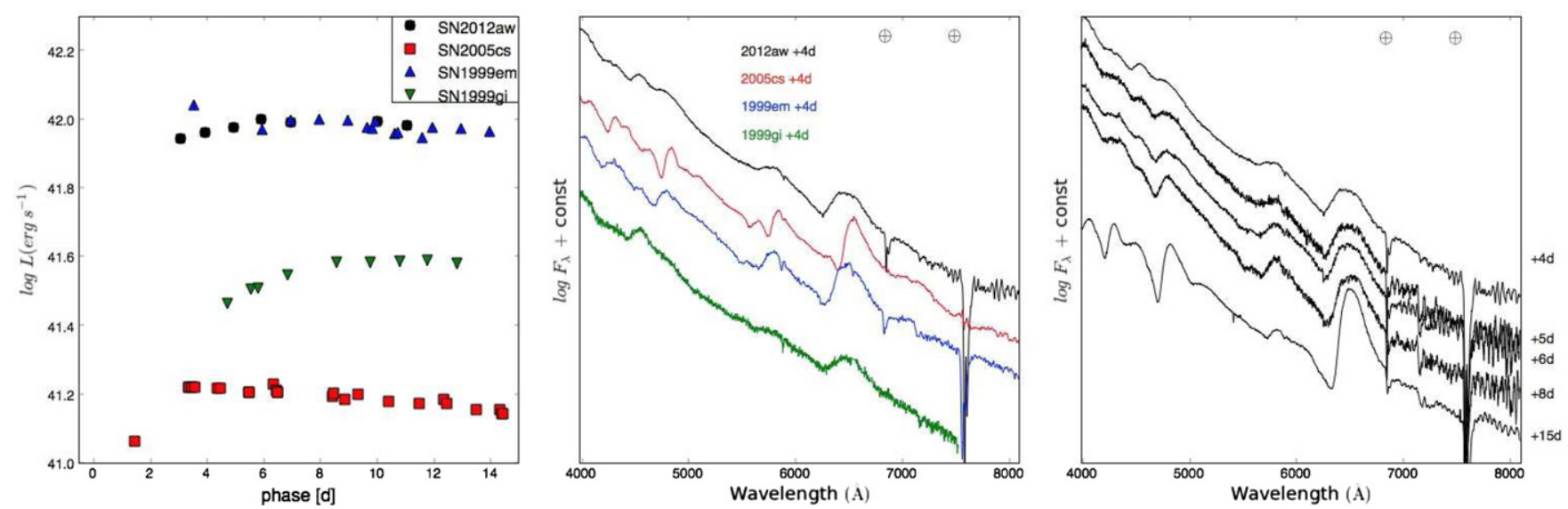

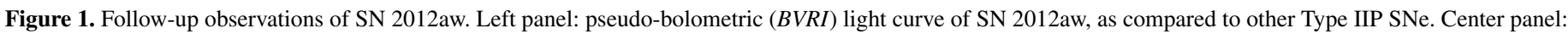

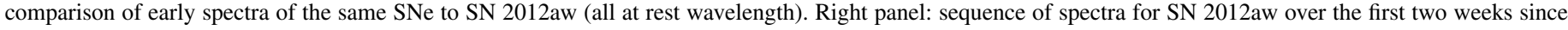
discovery.

(A color version of this figure is available in the online journal.)

circumstellar material. SN 2012aw was also detected in radio observations (Yadav et al. 2012; Stockdale et al. 2012) with a flux at $22 \mathrm{GHz}$ of $2 \times 10^{25} \mathrm{erg} \mathrm{s}^{-1} \mathrm{~Hz}^{-1}$ at +1 week after explosion, rising to $4 \times 10^{25} \mathrm{erg} \mathrm{s}^{-1} \mathrm{~Hz}^{-1}$ at +2 weeks. These values are similar to those of the sample of Type IIP $\mathrm{SNe}$ presented by Chevalier et al. (2006).

\section{SUPERNOVA CHARACTERIZATION AND FOLLOW-UP}

Our collaboration commenced an intensive spectroscopic and photometric follow-up campaign for SN 2012aw immediately after the discovery. Full coverage of the $\mathrm{SN}$ will be published in a future paper; here we present a limited set of optical observations from first two weeks after explosion to characterize the initial $\mathrm{SN}$ evolution.

Photometry was obtained for SN 2012aw with the Asiago Observatory Schmidt telescope in the Landolt BVRI system. The data were pipeline reduced, and point spread function (PSF)-fitting photometry was performed on the images using the QUBA pipeline (Valenti et al. 2011). A pseudobolometric (i.e., $B V R I$ ) light curve was constructed by integrating the observed SN flux over the optical filters. The resulting light curve is shown in Figure 1, together with those of three other Type IIP SNe for comparison (SN 1999em, Elmhamdi et al. 2003; SN 1999gi, Leonard et al. 2002; SN $2005 \mathrm{cs}$, Pastorello et al. 2009). The luminosity of SN 2012aw is comparable to that of the SN $1999 \mathrm{em}$, placing it firmly in the continuum of normal Type IIP SNe, as opposed to sub-luminous events such as SN 2005cs.

Spectra of SN 2012aw were obtained from the Asiago $122 \mathrm{~cm}$ telescope + Boller\&Chivens Spectrograph $+300 \mathrm{tr} / \mathrm{mm}$, and the Nordic Optical Telescope + ALFOSC + Gr\#4. Spectra were reduced, extracted, and flux and wavelength calibrated with the QUBA pipeline. The sequence of spectra from the first two weeks after explosion is presented in Figure 1. Without any adjustment for either Milky Way or host galaxy extinction, the slope of the SN 2012aw spectrum (epoch 3.9 days) is almost identical to that of SN 1999em at +4 days (Baron et al. 2000; Hamuy et al. 2001). Baron et al. found that a synthetic spectrum fit with $T_{\mathrm{BB}}=11,000-13,000 \mathrm{~K}$ and an extinction of $E(B-V) \sim 0.05 \mathrm{mag}$ provides a good match to the continuum slope and $\mathrm{H}$ I lines. A similar result would necessarily be determined for SN 2012aw given its striking similarity to SN $1999 \mathrm{em}$. The $\mathrm{H} \alpha$ absorption minimum is at a velocity of $v_{\text {phot }} \sim 13,000 \mathrm{~km} \mathrm{~s}^{-1}$, again almost identical to the minimum for SNe 1999em and 1999gi. Figure 1 shows the early spectra of these three Type IIP SNe illustrating the similarity, also shown is the low-energy, faint Type IIP SN 2005cs (Pastorello et al. 2009) which has an $\mathrm{H} \alpha$ minimum at a much lower velocity of $6500 \mathrm{~km} \mathrm{~s}^{-1}$. This clearly shows that SN 2012aw is not a low energy explosion.

A high-resolution spectrum of SN 2012aw was obtained with the Telescopio Nazionale Galileo + Sarg on March 29. The two components of the Na I D doublet were detected at 5907.998, $5914.004 \AA$, which are consistent with the recessional velocity of M95. The equivalent width of the two components were 0.286 and $0.240 \AA$, respectively. From the empirical relation of Munari \& Zwitter (1997), we estimate $E(B-V)=0.10 \pm$ $0.05 \mathrm{mag}$ for SN 2012aw. We note, however, that even if dust has been photo-evaporated in the explosion (see Section 4), the Na I absorption should remain unchanged and hence these calibrations may systematically overestimate the extinction.

\section{PROGENITOR OBSERVATIONS AND DATA ANALYSIS}

\subsection{Archival Data}

We have searched both the HST and Spitzer Space Telescope archives, along with the publicly available archives of all the major ground-based observing facilities, for pre-explosion images covering the site of SN 2012aw. A $\log$ of all data used is given in Table 1. The deepest and highest resolution images of M95 found were from the Wide-Field and Planetary Camera 2 (WFPC2) on board the HST. The site of SN 2012aw was observed on multiple occasions between 1994 November and 1995 January with the $F 439 W, F 555 W$, and $F 814 W$ filters, and again in the $F 555 W$ filter in 1995 December. $H S T$ +WFPC2 observed M95 again in $F 336 W$ and $F 658 N$ on 2009 January 18. NTT+SOFI $\left(K_{S}\right)$ and VLT+ISAAC $\left(J_{S}\right)$ images of M95 were obtained from the ESO archive. A subset of the data used is shown in Figure 2.

All HST data were downloaded from the Multimission Archive at STScI. ${ }^{9}$ The individual WFPC2 $F 814 \mathrm{~W}$

\footnotetext{
9 http://archive.stsci.edu
} 

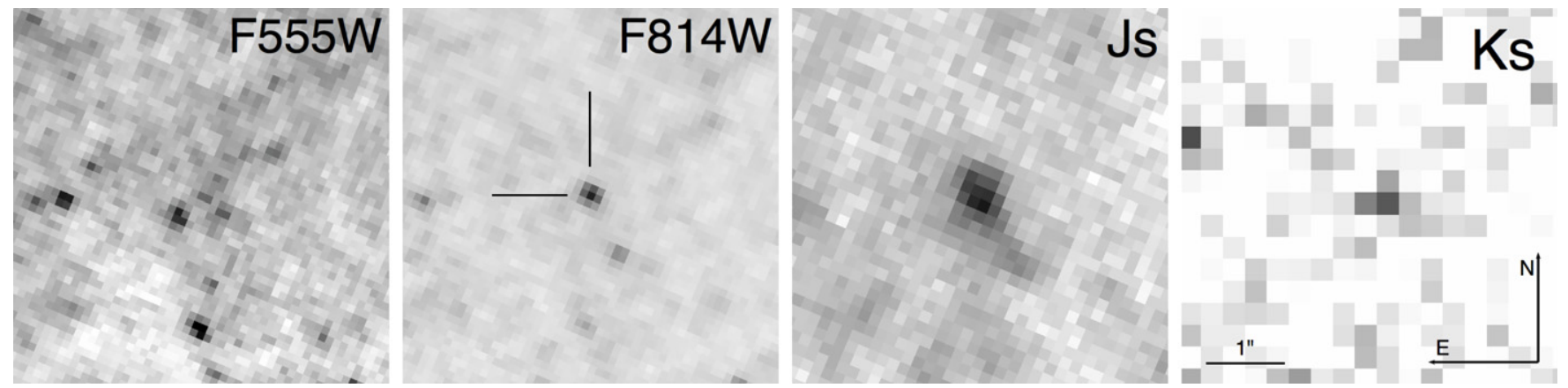

Figure 2. Progenitor of SN 2012aw as detected in archival pre-explosion images. Images are scaled and oriented as indicated and each panel is centered on the progenitor position as indicated with tick marks in the $F 814 \mathrm{~W}$ image. In the $J$-band image the progenitor is partially blended with a nearby source, this has been corrected for with PSF-fitting photometry.

Table 1

Pre-explosion Images of the Site of SN 2012aw

\begin{tabular}{lcccr}
\hline \hline Date & Telescope & Instrument & Filter & $\begin{array}{c}\text { Exposure } \\
(\mathrm{s})\end{array}$ \\
\hline 2009 Jan 18 & HST & WFPC2 & $F 336 W$ & 4400 \\
1994 Nov 29-1994 Dec 19 & $\ldots$ & $\ldots$ & $F 439 W$ & 5000 \\
2009 Jan 18 & $\ldots$ & $\ldots$ & $F 658 N$ & 1800 \\
1994 Nov 29-1995 Dec 4 & $\ldots$ & $\ldots$ & F555W & 34130 \\
1994 Nov 29-1995 Jan 16 & $\ldots$ & $\ldots$ & $F 814 W$ & 9830 \\
2000 Mar 26 & VLT & ISAAC & $J$ & 2400 \\
2000 Mar 27 & $\ldots$ & $\ldots$ & $\ldots$ & 4080 \\
2002 Mar 25 & NTT & SOFI & $K_{\mathrm{S}}$ & 600 \\
2006 Mar 24 & $\ldots$ & $\ldots$ & $\ldots$ & 600 \\
\hline
\end{tabular}

images were combined using the drizzle algorithm within IRAF ${ }^{10}$ (Fruchter \& Hook 2002), although as the offsets between the individual exposures were not in an optimal sub-pixel dither pattern, we did not resample the image to a smaller pixel scale. Small image shifts were removed using the TWEAKSHIFTS task to improve the quality of the alignment.

The individual VLT+ISAAC images were reduced (badpixel masked, flat-fielded, and sky-subtracted) using the ISAAC pipeline (version 6.0.6). The individual frames from each night where the SN position was in the field of view were then aligned with sub-pixel shifts and combined within IRAF. The image from the second night of ISAAC observations (2000 March 27) was used for all of the subsequent analysis, as the total on-source exposures are deeper, and the $\mathrm{SN}$ position is further from the edge of the chip. The NTT+SOFI data from 2006 March 24 were flat-fielded and sky-subtracted with the SOFI pipeline (version 1.5.4) before the individual on-source frames were combined. A total of $600 \mathrm{~s}$ was obtained on source, with an FWHM of 0".6. For the SOFI data from 2002 March 25, the SOFI pipeline was unable to reduce the data, and so the IRAF XDIMSUM package was used for the reduction.

The Spitzer Space Telescope+ IRAC observed M95 as part of the SINGS survey (Kennicutt et al. 2003), however, the spatial resolution of these data (1".7 PSF FWHM in the shortest wavelength channel) is ill suited for progenitor identification. We downloaded the IRAC data from the Spitzer Heritage Archive, but could not identify a point source at the SN coordinates. We have not considered the IRAC data any further in the following.

\footnotetext{
10 IRAF is distributed by the National Optical Astronomy Observatory, which is operated by the Association of Universities for Research in Astronomy, Inc., under cooperative agreement with the National Science Foundation.
}

\subsection{Progenitor Identification}

A deep, high-resolution $K$-band image of SN 2012aw was obtained on 2012 March 31 with Gemini+NIRI (Program: GN-2012A-Q-38). To match the resolution of the pre-explosion WFPC2 images, we used the ALTAIR adaptive optics system to correct for the seeing; as the SN was sufficiently bright (mag 13) at the time of the observations it was used as a natural guide star for ALTAIR. The f14 camera was used, which has a 0 .'05 pixel scale over a $51^{\prime \prime} \times 51^{\prime \prime}$ field of view. As the region around SN 2012aw is not crowded, we used on-source dithers of a few arcseconds and median combined these to create the sky frame for each exposure. The data were reduced with the IRAF GEMINI package, yielding a final combined image with a PSF FWHM of 0.2 .

Forty sources were identified common to both the NIRI and the drizzled WFPC2 $F 814 W$ images. The positions of these reference stars were measured in both images with IRAF PHOT. After rejecting outliers from the fit, which either had low signal to noise or centering errors, we used 22 sources to derive a geometric transformation between the pixel coordinate systems of the WFPC2 and NIRI images. A general fit allowing for scaling, a shift in $x$ and $y$, rotation and a skew term was used, with a root mean square error in the fit of 53 mas. The pixel coordinates of SN 2012aw were measured in the NIRI image and transformed to the pixel coordinates of the WFPC2 image. The transformed position of the SN coincided with the same source identified by Elias-Rosa et al. (2012) and Fraser et al. (2012). The position of this source was measured using the three different centering algorithms in РНОт, all of which agreed to within 14 mas. The separation between the transformed SN position and the progenitor candidate was 27 mas, which is well within the total (transformation $+\mathrm{SN}$ position + progenitor position) uncertainty of 55 mas. Hence, we formally identify the source as the progenitor candidate for SN 2012aw.

\subsection{Progenitor Photometry}

Photometry was performed on the WFPC2 images with the нSтPнот package (Dolphin 2000a), which is designed specifically for undersampled data from this instrument. The pipeline-reduced images were first masked for bad pixels, hot pixels, and cosmic rays using the ancillary packages distributed with HSTPHOT. After masking, PSF-fitting photometry was performed on the individual images, which typically had exposure times of 1000-2000 s. The SN progenitor candidate was detected at a magnitude of $F 555 \mathrm{~W}=26.70 \pm 0.06$ and $F 814 \mathrm{~W}=$ $23.39 \pm 0.02$ (in the photometric system as defined in Dolphin 2000b), but was not detected in either the $F 336 \mathrm{~W}$ 

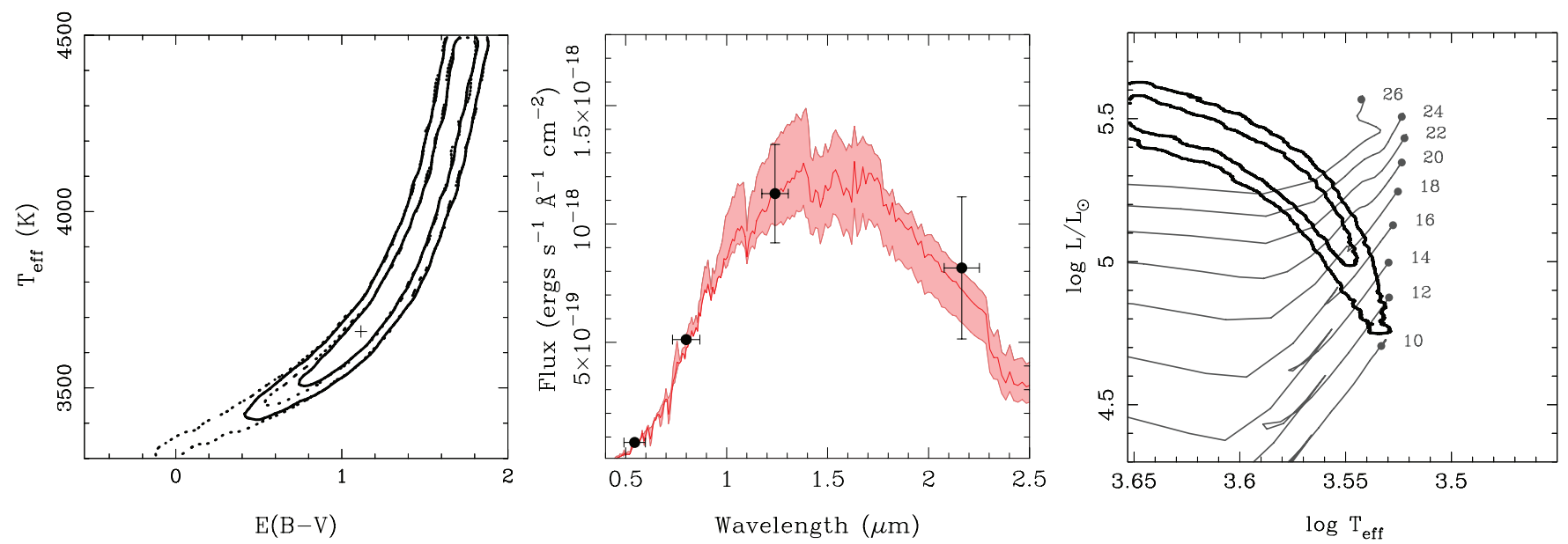

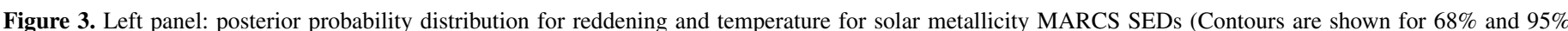

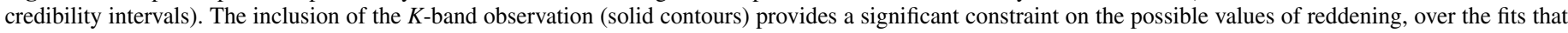

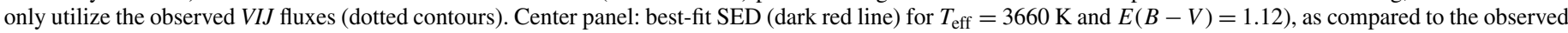

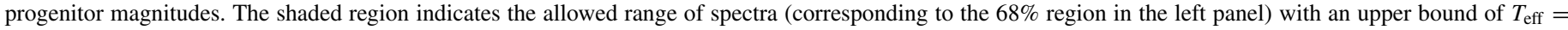

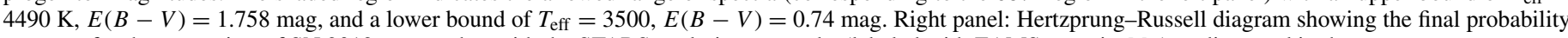
contours for the progenitor of SN 2012aw, together with the STARS evolutionary tracks (labeled with ZAMS mass in $M_{\odot}$ ) as discussed in the text.

(A color version of this figure is available in the online journal.)

or $F 439 W$ filter images. Based on photometry of sources detected by нотрнот in the $F 439 W$ image, we estimate a $5 \sigma$ limiting magnitude of $F 439 W>26$. The $F 814 W$ magnitude for the progenitor, as measured in the individual frames, appears to be unchanged within the uncertainties over the $\sim 50$ day period from the first to the last observation. Unfortunately, the progenitor is too faint to check for variability in the $F 555 \mathrm{~W}$ images. The $F 658 N(\mathrm{H} \alpha)$ WFPC2 image was also examined, but there was no sign of emission at the $\mathrm{SN}$ position.

The SOFI $K_{S}$ and ISAAC $J_{S}$ images were aligned to an $F 814 W$-filter mosaic of the four WFPC2 chips, and a counterpart to the optical progenitor candidate was found in both images. PSF-fitting photometry was performed on the ISAAC $J_{\mathrm{s}}$ and SOFI $K_{\mathrm{s}}$ image with the SNOOPY package in IRAF. A magnitude was found for the progenitor of $J=21.1 \pm 0.2$ (setting the photometric zero point from the $J$ magnitude of two Two Micron All Sky Survey (2MASS) sources in the field, which is the dominant source of error). As the bandpasses of the $J_{\mathrm{s}}$ and $J$ filters are different, we calculated color terms from synthetic photometry of MARCS model spectra between 3000 and $4000 \mathrm{~K}$. Over this temperature range the color term is $\lesssim 0.02 \mathrm{mag}$, which is significantly less than our photometric error. For the SOFI $K_{\mathrm{s}}$ image from 2006, we measured a magnitude of $K=19.4 \pm$ 0.4 with PSF-fitting photometry. The uncertainty in the measurement consists of 0.36 mag from the progenitor photometry, and 0.19 uncertainty in the zero point, which was set from six 2MASS sources in the field. As a check of the PSF-fitting measurement, aperture photometry was performed on the same image with IRAF phot, which returned a magnitude of $K=19.1 \pm$ 0.4 . Aperture photometry of the SOFI image from 2002 yielded a magnitude for the progenitor of $18.9 \pm 0.3 \mathrm{mag}$. We hence adopt an average value of $K=19.1$ from the two epochs for the progenitor magnitude, with a conservative error of \pm 0.4 mag. All NIR magnitudes are in the 2MASS system (Cohen et al. 2003).

We note that a more detailed analysis by Van Dyk et al. (2012) found comparable magnitudes for the progenitor of SN 2012aw, but with smaller uncertainties in the NIR through the use of an improved photometric calibration.

\section{RESULTS AND DISCUSSION}

We used our own Bayesian spectral energy distribution (SED) fitting code (based on the Bayesian Inference-X nested sampling framework; J. R. Maund 2012 in preparation; Skilling 2004) to compare the observed progenitor magnitudes to synthetic photometry of MARCS model spectra (Gustafsson et al. 2008). The models used were for $15 M_{\odot}$ stars with $\log g=0$ dex and solar metallicity, with temperatures between 3300 and $4400 \mathrm{~K}$. The SEDs were rebinned by a factor of 10 prior to the calculation of synthetic photometry (Plez 2008). We fit the progenitor VIJK magnitudes with flat priors on $T_{\text {eff }}$ and $E(B-V)$, which was allowed to vary between $-0.5<E(B-V)<2$. A match was found with the observed progenitor magnitudes along a banana-shaped region as shown in Figure 3.

The luminosity is ill constrained due to the uncertain extinction, and indeed varies within the $1 \sigma$ contours between 5.0 dex at $3550 \mathrm{~K}$ to $5.6 \mathrm{dex}$ for a progenitor with $T_{\text {eff }}=4450 \mathrm{~K}$. The SED fit is poorer in $K$ than the $J$ or WFPC 2 data, as can be seen in Figure 3. Besides the larger photometric errors in $K$ data, the models are also quite sensitive to metallicity at cooler temperatures, due to the $\mathrm{TiO}$ absorption which is present in the NIR spectra of cool stars. Determining the luminosity of the progenitor from the $K$-band magnitude only (as $A_{K} \sim 0.1 A_{V}$, and the bolometric correction to $K$ band only changes by 1.1 mag between 3300 and $4500 \mathrm{~K}$ ) gives a value between 4.9 and 5.5 dex. We also attempted to fit the progenitor SED with MARCS spectra of twice solar metallicity models, but found the fit to be poorer than for the solar metallicity models.

In Figure 3, we have also plotted stellar evolutionary tracks from the STARS code (Eldridge et al. 2008). Comparing the luminosity of the progenitor to the endpoints of the tracks implies a zero-age main-sequence (ZAMS) mass of between 14 and $26 M_{\odot}$. If we consider the hotter progenitors in Figure 3, the discrepancy in temperature with the endpoints of the STARS evolutionary tracks becomes more apparent. However, increased mass loss, either in a binary or through rotation, would serve to bring the endpoints of these tracks over to hotter temperatures (Georgy 2012). 
As the radius of the progenitor can be expressed as $R=$ $\sqrt{L / T_{\text {eff }}^{4}}$, it is easy to calculate the expected radius for each point in the HR diagram. We find that even for a temperature of $4500 \mathrm{~K}$, and a luminosity of $5 \mathrm{dex}$, the radius of the progenitor is still $>500 R_{\odot}$. This is sufficiently large that we may reasonably expect the progenitor to give rise to a Type IIP SN (e.g., Popov 1993; Kasen \& Woosley 2009), and so cannot be used to further restrict the region of the HRD where the progenitor lies.

Within the $68 \%$ confidence contours, the solar metallicity SEDs favor an extinction which is greater than $E(B-V)>0.8$ mag, although within $95 \%(2 \sigma)$, there is a solution with a correspondingly poorer fit for $E(B-V)=0.4 \mathrm{mag}$. The implication of this is that a significant amount of dust could have been destroyed in the initial phases of the SN explosion (e.g., Waxman \& Draine 2000; Dwek et al. 2008), and that the extinction toward an SN cannot be taken as a proxy for the extinction toward the progenitor. We stress, however, that regardless of extinction, SN 2012aw potentially arises from one of the highest mass progenitors found to date.

As was recently suggested by Walmswell \& Eldridge (2011), significant amounts of circumstellar dust around SN progenitors is an appealing solution to the lack of high-mass red supergiant progenitors identified by Smartt et al. (2009). We caution, however, that SN 2012aw is the reddest SN progenitor found thus far, and appears to suffer from extinction that is comparable to that of the most luminous Galactic red supergiants (Levesque et al. 2005), but higher than is typical for a Type IIP progenitor (from the limited sample with color information). Hence, it remains unclear whether circumstellar dust truly is the panacea for the "red supergiant problem."

We also note that based on a preprint of this paper, Kochanek et al. (2012) suggested that we may overestimate the luminosity of the progenitor of SN 2012aw. Kochanek et al. suggest that assuming an average dust composition, with $R_{V}=3.1$, is likely incorrect for an individual $\sim 20 M_{\odot}$ red supergiant which will likely have silicate dust. Furthermore, some of the stellar light scattered by dust will be re-emitted at optical wavelengths. Their more detailed modeling of circumstellar material dust around the progenitor yields progenitor luminosity of $<5 \mathrm{dex}$, at the lower extremum of our uncertainty range.

We acknowledge funding from STFC (M.F.), the ERC (S.J.S.), and the Royal Society (J.R.M.). S.B., M.T., I.J.D., and A.P. are partially supported by the PRIN-INAF 2009 with the project "Supernovae Variety and Nucleosynthesis Yields."

Based on observations obtained at the Gemini Observatory, and on observations made with the NASA/ESA Hubble Space Telescope, obtained from the data archive at the STScI.

Based on data obtained from the ESO Science Archive Facility. Partially based on observations from Asiago observatory, Galileo 1.22 and Schmidt 67/92 telescopes operated by Padova University and INAF OAPd; and the Nordic Optical Telescope, operated jointly by Denmark, Finland, Iceland, Norway, and Sweden, in the Spanish Observatorio del Roque de los Muchachos of the Instituto de Astrofisica de Canarias.
We thank Ben Davies and Rolf Kudritzki for useful advice on metallicities, Valentin Ivanov for comments on SOFI reductions, and Christopher Kochanek for discussions on extinction.

Facilities: Gemini:Gillet (NIRI, ALTAIR), HST (WFPC2), VLT:Melipal (ISAAC), NTT (SOFI)

\section{REFERENCES}

Baron, E., Branch, D., Hauschildt, P. H., et al. 2000, ApJ, 545, 444 Chevalier, R. A., Fransson, C., \& Nymark, T. K. 2006, ApJ, 641, 1029

Cohen, M., Wheaton, Wm. A., \& Megeath, S. T. 2003, AJ, 126, 1090

Dolphin, A. E. 2000a, PASP, 112, 1383

Dolphin, A. E. 2000b, PASP, 112, 1397

Dwek, E., Arendt, R. G., Bouchet, P., et al. 2008, ApJ, 676, 1029

Eldridge, J. J., Izzard, R. G., \& Tout, C. A. 2008, MNRAS, 384, 1109

Elias-Rosa, N., Van Dyk, S. D., Cuillandre, J. C., Cenko, S. B., \& Filippenko, A. V. 2012, ATel, 3991

Elmhamdi, A., Danziger, I. J., Chugai, N., et al. 2003, MNRAS, 338, 939

Fagotti, P., Dimai, A., Quadri, U., et al. 2012, CBET, 3054, 1

Fox, D. W., Lewin, W. H. G., Fabian, A., et al. 2000, MNRAS, 319, 1154

Fraser, M., Ergon, M., Eldridge, J. J., et al. 2011, MNRAS, 417, 1417

Fraser, M., Maund, J. R., Smartt, S. J., et al. 2012, ATel, 3994

Freedman, W. L., Madore, B. F., Gibson, B. K., et al. 2001, ApJ, 553, 47

Fruchter, A. S., \& Hook, R. N. 2002, PASP, 114, 144

Georgy, C. 2012, A\&A, 538, 8

Gustafsson, B., Edvardsson, B., Eriksson, K., et al. 2008, A\&A, 486, 951

Hamuy, M., Pinto, P. A., Maza, J., et al. 2001, ApJ, 558, 615

Hunter, I., Brott, I., Langer, N., et al. 2009, A\&A, 496, 841

Immler, S., \& Brown, P. J. 2012, ATel, 3995

Itoh, R., Ui, T., \& Yamanaka, M. 2012, CBET, 3054, 2

Kasen, D., \& Woosley, S. E. 2009, ApJ, 703, 2205

Kennicutt, R. C., Jr., Armus, L., Bendo, G., et al. 2003, PASP, 115, 928

Kochanek, C. S., Khan, R., \& Dai, X. 2012, ApJ, submitted

Leonard, D. C., Filippenko, A. V., Li, W., et al. 2002, AJ, 124, 2490

Levesque, E. M., Massey, P., Olsen, K. A. G., et al. 2005, ApJ, 628, 973

Maund, J. R., \& Smartt, S. J. 2009, Science, 324, 486

Mattila, S., Smartt, S. J., Eldridge, J. J., et al. 2008, ApJ, 688, 91

McCall, M. L., Rybski, P. M., \& Shields, G. A. 1985, ApJS, 57, 1

Munari, U. 2012, CBET, 3054, 3

Munari, U., \& Zwitter, T. 1997, A\&A, 318, 269

Pastorello, A., Valenti, S., Zampieri, L., et al. 2009, MNRAS, 394, 2266

Pettini, M., \& Pagel, B. E. J. 2004, MNRAS, 348, 59

Pilyugin, L. S., Thuan, T. X., \& Vílchez, J. M. 2006, MNRAS, 367, 1139

Plez, B. 2008, in Astronomical Spectroscopy and Virtual Observatory, ed. M. Guainazzi \& P. Osuna (Villafranca del Castillo: European Space Astronomy Centre), 169

Popov, D. V. 1993, ApJ, 414, 712

Poznanski, D., Nugent, P. E., Ofek, E. O., Gal-Yam, A., \& Kasliwal, M. M. 2012, ATel, 3996

Rizzi, L., Tully, B., Makarov, D., et al. 2007, ApJ, 661, 815

Schlegel, D. J., Finkbeiner, D. P., \& Davis, M. 1998, ApJ, 500, 525

Siviero, A., Tomasella, L., Pastorello, A., et al. 2012, CBET, 3054, 4

Skilling, J. 2004, in AIP Conf. Ser. 735, Bayesian Interference and Maximum

Entropy Methods in Science and Engineering, ed. R. Fischer, R. Preuss, \& U. V. Toussaint (Melville, NY: AIP), 395

Smartt, S. J., Eldridge, J. J., Crockett, R. M., \& Maund, J. R. 2009, MNRAS, 395, 1409

Smartt, S. J., Maund, J. R., Hendry, M. A., et al. 2004, Science, 303, 499

Stockdale, C. J., Ryder, S. D., Van Dyk, S. D., et al. 2012, ATel, 4012

Walmswell, J. J., \& Eldridge, J. J. 2011, MNRAS, 419, 2054

Waxman, E., \& Draine, B. T. 2000, ApJ, 537, 796

Valenti, S., Fraser, M., Benetti, S., et al. 2011, MNRAS, 416, 3138

Van Dyk, S. D., Cenko, S. B., Poznanski, D., et al. 2012, ApJ, 756, 131

Van Dyk, S. D., Li, W., \& Filippenko, A. V. 2003, PASP, 115, 1289

Yadav, N., Chakraborti, S., \& Ray, A. 2012, ATel, 4010 